\title{
Comparison of Sound Velocity Estimation and Classification Methods for Ultrasonic Testing of Cheese
}

\author{
I. KERTÉSZ ${ }^{*}$, J. FELFÖLDI ${ }^{1}$
}

\begin{abstract}
Testing of two methods novel to ultrasonic measurements was carried out on cheese samples to estimate the Time-of-Flight (TOF) parameter. The Short Time Average /Long Time Average (STA/LTA) method and the Autoregressive Akaike Information Criterion Picker (AR-AIC picker) method are used mainly in seismology for earthquake event detection. The STA/LTA method proved to be ineffective with such noise level that is present during ultrasonic measurements, but the AIC picker algorithm yielded reliable results. A new approach for classification was tested on two types of samples, those were matching in composition, but different in treatment and texture. The method used is based on the results of wavelet decomposition, and after retrieving sufficient spectral data, a linear discriminant analysis (DA) resulted in $100 \%$ correct classification, which was compared to the DA classification results based on other methods.
\end{abstract}

Keywords: ultrasonic, non-destructive testing, NDT, Time of Flight, STA/LTA, AIC

The industrial use of testing (passive) ultrasounds is spreading continuously, although active ultrasounds are more prominent among applied technologies. As for texture analysis application, to assess quality attributes MIZRACH and co-workers used ultrasonic testing with fruits such as avocado and plum (MIZRACH et al. 1996, 1999, 2004), and BENEDITO and co-workers used the method for cheese quality and maturity estimation in several cases (BENEDITO et al. 2000a, 2000b, 2006). Most such studies often yield good results, but barely use signal forms different from simple sine waves. Using sine waves exclusively makes spectral testing a tedious work, and allows use of only a few frequencies, as in the study of LÉTANG and coworkers (2001) and CORREDIG and co-workers (2004). Other signal types have been designed in order to conduct such tests efficiently. One of these

* Corresponding author. E-mail: istvan.kertesz@uni-corvinus.hu

1Szent István University, Faculty of Food Science, Department of Physics and Control, Somlói út 14-16, H-1118 Budapest, Hungary 
signal forms is the so-called "chirp" signal, which has been applied successfully according to certain papers. Using a chirp signal, HÄUPLER and co-workers (2014) reported a good connection between the fat content of cocoa butter and attenuation. In another paper, LEEMANS and DESTAIN (2009) developed a method for foreign body detection with chirp signals.

The authors of this paper previously conducted storage experiments based on the studies of BENEDITO and co-workers (BENEDITO et al. 2000a) for following changes during maturation and to distinguish cheeses with different fat content, which is a major factor affecting attenuation of sound signals. This paper shows results of experiments carried out on two cheeses of the same composition, the difference is only in the treatment, and conclusively the texture.

\section{Materials and Methods}

The cheeses investigated were commercially available sliced mozzarellas (Szarvasi Mozzarela Kft., Hungary), purchased in a $100 \mathrm{~g}$ tray packaging, each package containing ten slices. Fourty slices of plain, untreated, and twenty slices of smoked cheeses were investigated with ultrasonic testing. The thickness, which is the most influential parameter for measuring the TOF, showed minor differences and was not found to be significant $(\mathrm{p}=$ $0.062, \alpha=0.05$, overall average of thickness $=2.12 \mathrm{~mm}$ ). Composition of the two sample types were matching, only the treatment was the difference between them. There was a sensible difference in texture, but this was not quantified by measurement, therefore not taken into account as a factor for prediction or verification.

The experimental setup (Fig. 1) consisted of a piezoelectric transducer couple (an interchangeable pulser-receiver couple) with a nominal frequency of $250 \mathrm{kHz}$, a Velleman PCSGU250 function generator and oscilloscope, and a PC to save the measurement files in ASCII format. Ultrasonic gel was applied between the samples and the transducers for better acoustic coupling. 


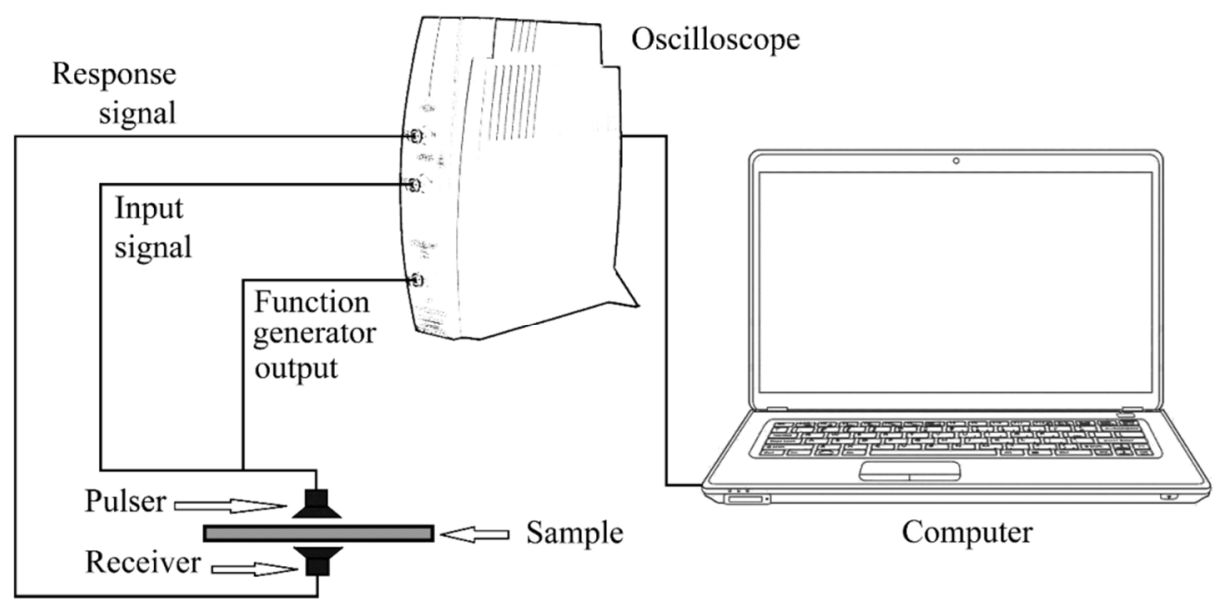

Fig. 1. Experimental setup

The applied signal form was the chirp signal, which is a sine wave of constantly increasing frequency from 50 to $450 \mathrm{kHz}$, and is modulated by a Hanning envelope. This signal form is double modulated, which includes continuous amplitude and frequency modulation. This enables better detection of different frequency-related behaviour of the transmission through the measured media. As opposed to measurements conducted with a series of fixed frequency sine waves, the use of a chirp signal results in a detection more rapid by several orders of magnitude, which is vital for faster overall signal processing, and at the same time allows better spectral resolution. An example of the input and the response signals is shown in Fig. 2. A program was developed in Matlab R2012a, in which the algorithms

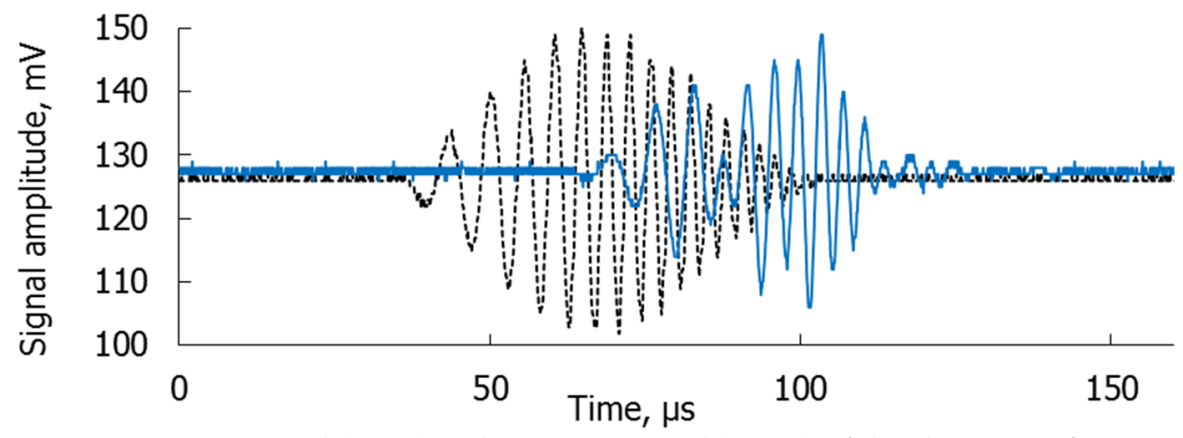

Fig. 2. Input signal (----) and response signal (__ ) of the chirp waveform 
for STA/LTA, AIC-picker and the wavelet analysis were written. Before further processing, the signals were de-noised with a wavelet-based built-in algorithm. Discriminant analyses were conducted in IBM SPSS Statistics 20.

The methods utilized for estimation of the Time-of-Flight (TOF) parameter - the time elapsed between the two signals - were the STA/LTA and the AR-AIC picker methods, both of which are known from the field of seismology and none of them were used in the food industry to date.

\subsection{STA/LTA method}

The STA/LTA estimator is very sensitive regarding parameterization, therefore requires minute fine-tuning (TRNKOCZY 2012). Two windows of different lengths are run through the signal, within the windows values of data points are averaged, then the two new datasets are divided by each other. The algorithm is simple, as shown in equation 1 :

$$
a_{i}=\frac{\sum_{j=i-s w}^{i} x_{j}}{s w} / \frac{\sum_{j=i}^{i+l w} x_{j}}{l w}
$$

where $a_{i}$ is the ratio of the two windows' average, $x_{j}$ is the series representing the signal units of which in our case is $\mathrm{mV}, i$ is the time index, $s w$ and $l w$ are the length of the short and long windows, respectively, both counted in number of data points. In the original methodology of the estimator, a trigger level has to be calculated to eliminate the disturbances in the result caused by noise and to reliably calculate the arrival of the signal. This is nearly impossible for the received signals in this study, therefore the peak values of the original and response signals were evaluated. The reason why this can be carried out is that it is not necessary to know the exact arrival of the signal, only the difference of the calculated arrivals, which is the only variable that can be extracted reliably (Fig. 3). The most problematic part of using the STA/LTA estimator is determining the optimum window lengths. Since the effect of their length compared to each other is not trivial and difficult to assess (especially for noisy signals), a stepwise optimization is required, which was also carried out by the program. The output variable was only the TOF, calculated as the distance between the estimators' peaks, but for the individual measurements nonuniform window lengths were allowed, the lengths of these were only matching in case of input and response signal couples. It is important to mention that the term 'noisy' does not refer to high amplitude noise per se, 
the noise level is a property that is determined by the final STA/LTA function, and is relative to the level of STA/LTA values at the actual recognizable signal shape (Fig. 3). The division of the two windows' average
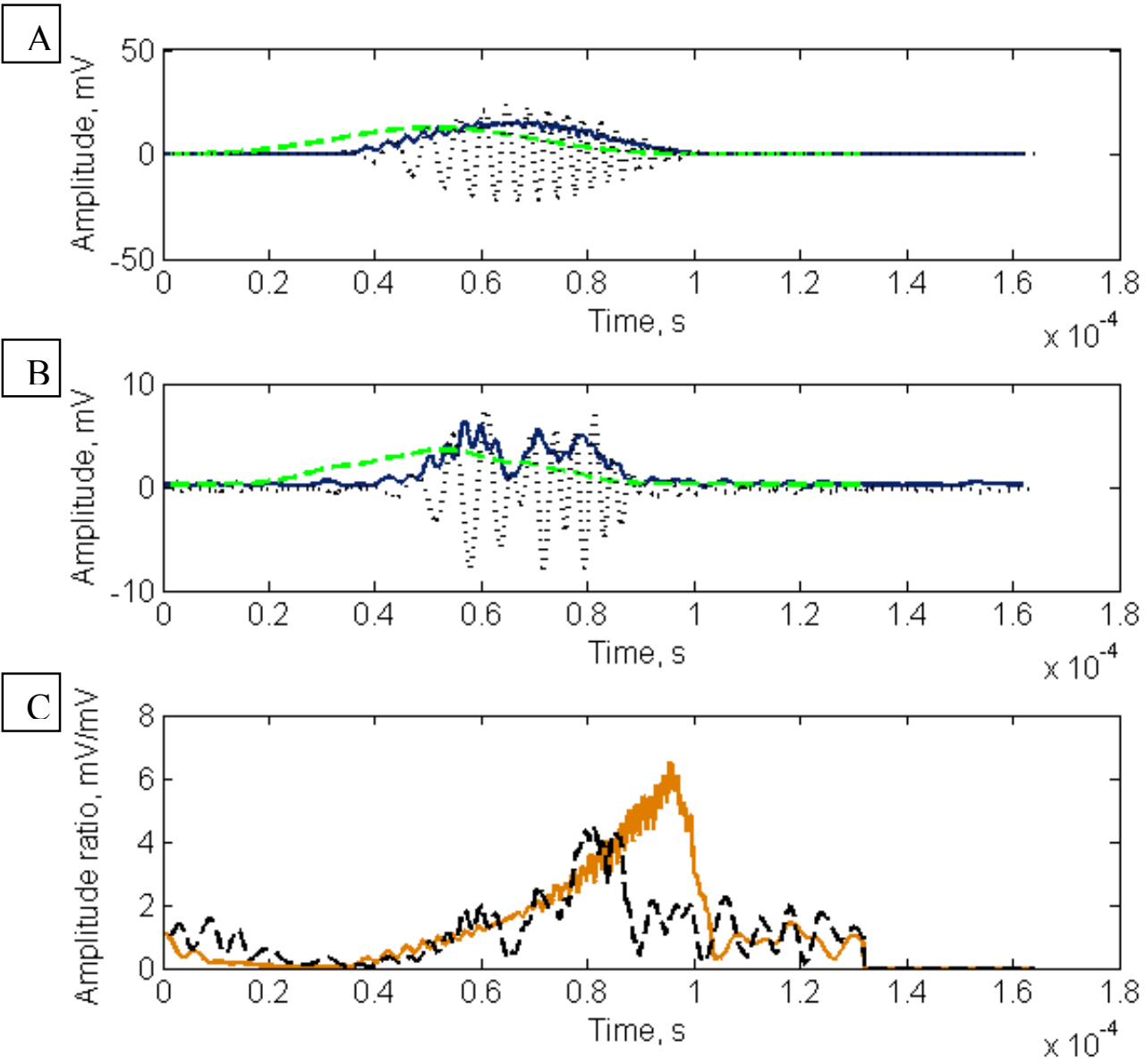

Fig. 3. Original signals $(\cdots \cdots .$.$) , their STA (\longrightarrow$ ) and LTA $(\longrightarrow$ functions (window sizes: 50 and 400 points, respectively) for the input signal (A) and the response signal (B). The final STA/LTA functions of the input $(-)$ and response (----) signals (C)

values causes high fluctuations in the final STA/LTA value in case of small, but sudden disturbances in the signal, which can be countered by applying a longer window. The implication of the effort to smooth out these bumps in the signal is that the two windows' lengths become too similar and the 
ratio of the averages too small and close to uniform in figure, making it inapt for use.

\subsection{AR-AIC picker method}

The AIC picker is a more sophisticated method, which is based on detecting the differences in variance throughout the characteristic function $(\mathrm{CF})$ of the signal, and its minimum indicates the arrival of the signal. The equation proposed by SEDLAK and co-workers (2008) is the following:

$A I C(k)=k \cdot \log (\operatorname{var}[C F(1 . . k)])+(N-k-1) \cdot \log (\operatorname{var}[C F(\{k+1\} \ldots N)])$

where $k$ is the $\mathrm{k}^{\text {th }}$ element of the $\mathrm{CF}, N$ is the number of elements of the CF. This formula implies that again two windows are applied, in this case to the $\mathrm{CF}$, but the size of these are gradually changing over time and do not require optimization, meaning a lower processing time. The CF in this study was calculated by the following equation suggested by ALLEN (1982):

$$
C F(i)=y(i)^{2}+K(y(i)-y(i-1))^{2}
$$

where $K$ is an empirical amplifier value, $y(i)$ is the $i^{\text {th }}$ element of the original signal $\mathrm{y}$. The parameters used were $K=1$ and $K=25$ for every measurement. According to SEDLAK and co-workers, a modified formulation (the use of absolutes instead of squared values) emphasizes the effect of differing frequencies in the $\mathrm{CF}$, which would be important to distinguish the signal from the noise, but the benefits of this effect were not observed for our signals; therefore, the original formula was used.

The problem with the AIC picker is that it is designed to indicate the arrival of the signal and does not describe its spread (Fig. 4), which may be an important indicator to evaluate the acoustic dispersion and conclusively the inhomogeneity of the examined sample. Depending on the goal, this may not be necessary, for classification purposes we do not need one exact number to characterize it. Although the STA/LTA method theoretically can be used to estimate the spread, the AIC function does not show apparent characteristics in respect to the original signal after the minimum. 


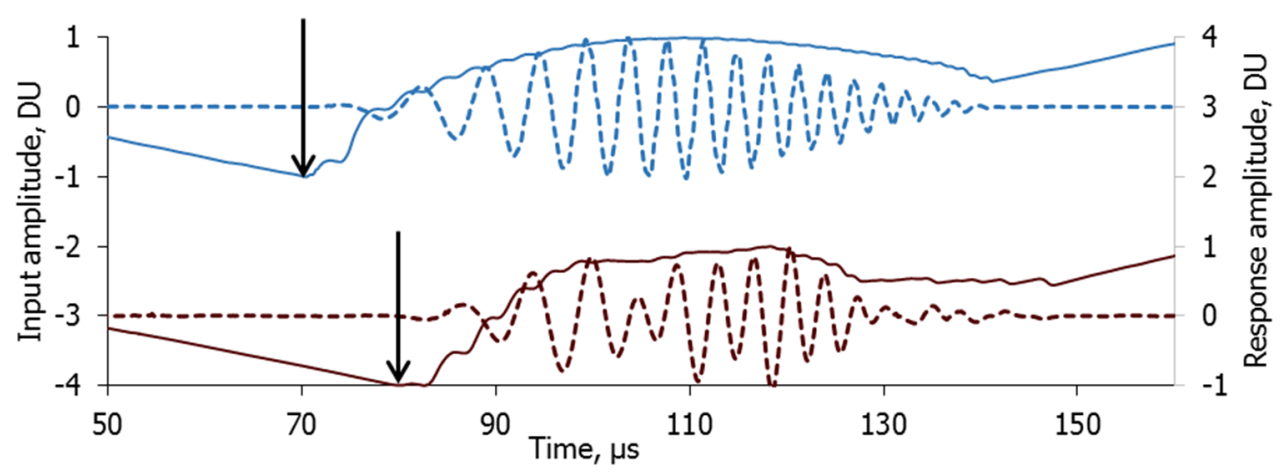

Fig. 4. Input (---) and response (----) signals and the corresponding AIC functions

( $\_$for input $\mathrm{AIC},-$ for response AIC), arrows mark their minima

\subsection{Classification by wavelet-decomposition}

For classification by cheese type, a linear discriminant analysis (LDA) of the results of a wavelet-based method was carried out and compared to other estimators. Wavelet decomposition was done with Daubechies wavelet (DAUBECHIES 1994) at refinement level 8 (db8). The wavelet function was chosen with respect to retaining the original frequencies and to maximize the differences between the wavelet coefficients of the maxima and minima of the waves, which is key to increase the effectiveness of the DA. The wavelet decomposition was carried out on the dataset of the crosscorrelation of the input and response signals. The resulting matrix of wavelet coefficients was analysed to extract the time of occurrence of the global maxima of every frequency of the wavelet analysis. This method grants a significantly lower amount of variables than if all the data points were analysed (65 instead of over 200,000 per signal), causing the processing time to be remarkably lower. The 'scale' is the factor of multiplication for the length of the wavelet, which is then cross-correlated with the signal for every multiplier, therefore, it corresponds to the wavelength. The original signals and the scalograms of the wavelet decompositions are shown in Fig. 5. 

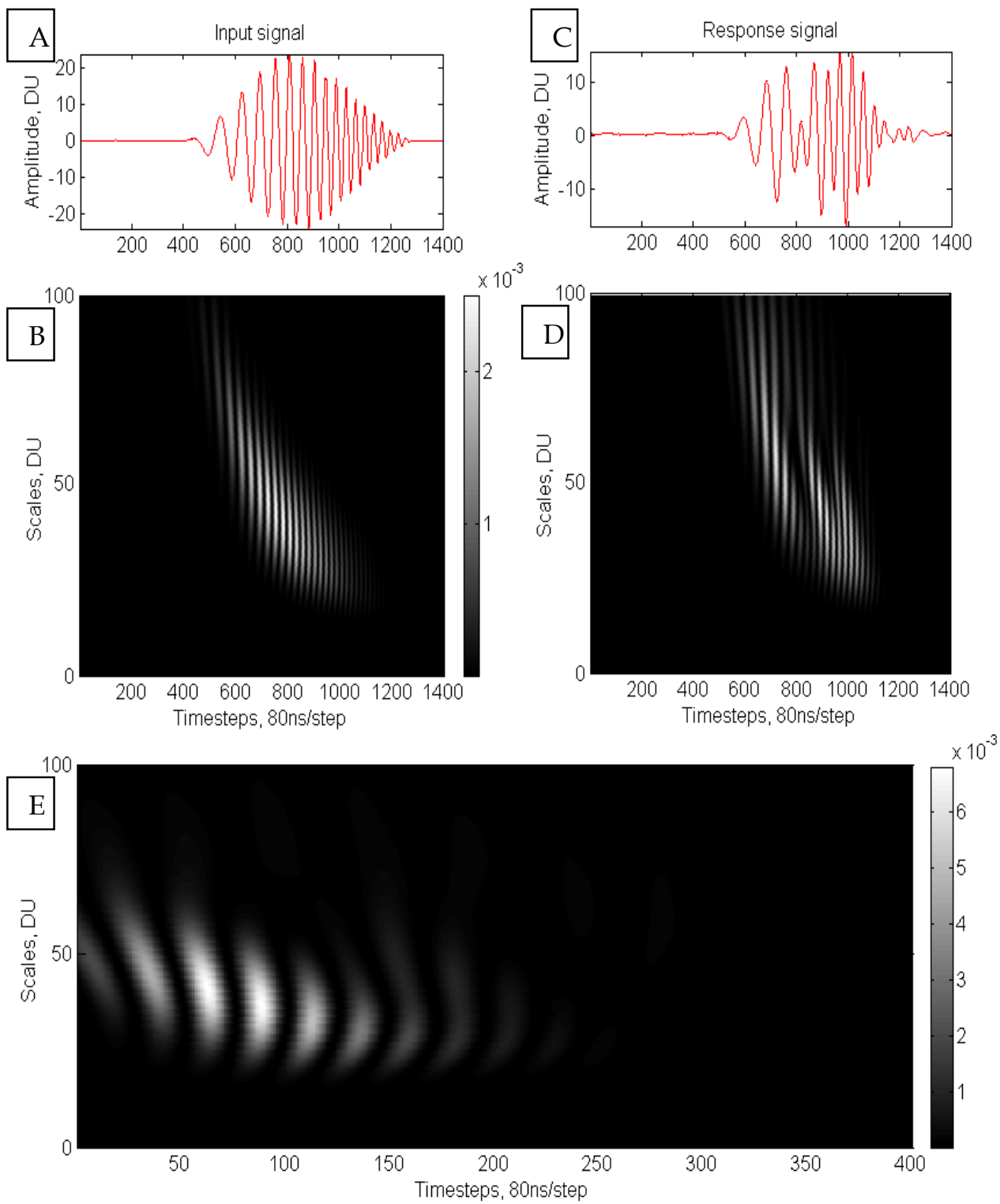

Fig. 5. The input signal (A) and its scalogram (B), the response signal (C) and its scalogram (D) and the scalogram of the cross-correlation function of the input and output signals (E), the colour scale shows the percentage of the signal's total energy for each wavelet 
The most important feature of this method is that it carries information about not only the spread of the signal, but also the spread of every examinable frequency defined by the resolution (pre-set value).

\section{Results and Discussion}

The STA/LTA method showed unrealistic (in several cases negative) values, regardless of the parameters used. This is probably because it was designed for earthquake detection, and therefore it is not reliable at such high noise levels as what is present in the conducted experiments. The results showed a high variance and no apparent tendency in the positive interval. The mean and standard deviation of the maximum values connected to the best pair of windows (which were not uniform for the individual signals for the best results) were $16.5 \mu \mathrm{s}$ and $14.3 \mu \mathrm{s}$, respectively, with a median of $7.84 \mu \mathrm{s}$. This shows the obvious inconsistency of results and because a stepwise optimization process is needed to achieve the optimum window sizes, the average processing time became unreasonably large (17.7 s).

On the other hand, AIC picker was found to be robust and yielded reliable values for the TOF, with a mean of $9.1 \mu$ s and a standard deviation of $1.46 \mu \mathrm{s}$, which are slightly higher than expected, but realistic for the samples in question. The average processing time of individual signals (despite of the complexity of the algorithm) was $0.77 \mathrm{~s}$, which is suitable for industrial purposes. In earlier attempts on estimating the TOF, the maximum of cross-correlated signals was found to be a suitable estimator, but in the present experiment, it yielded unsatisfying results. In Fig. 6(a), it is shown that the cross-correlation estimator created two distinct groups of TOF values, and the groups are not corresponding with the sample types. Therefore -even though the standard deviation is lower than that of the AIC picker's results - it is not reliable, and the AIC picker's more balanced estimations (as shown in Fig. 6(b)) should be used further.

Wavelet analysis proved to be very helpful for classification of the samples by type. As a point of reference, the same linear DA was carried out with different predictor variables, the classification matrices are shown in Tables 1-3. When thickness and TOF values were used as input variables, the two factors in the calculation of sound velocity, which corresponds to elasticity, the ratio of correctly classified samples was $71.7 \%$ (crossvalidation $[\mathrm{CV}]: 66.7 \%)$. 

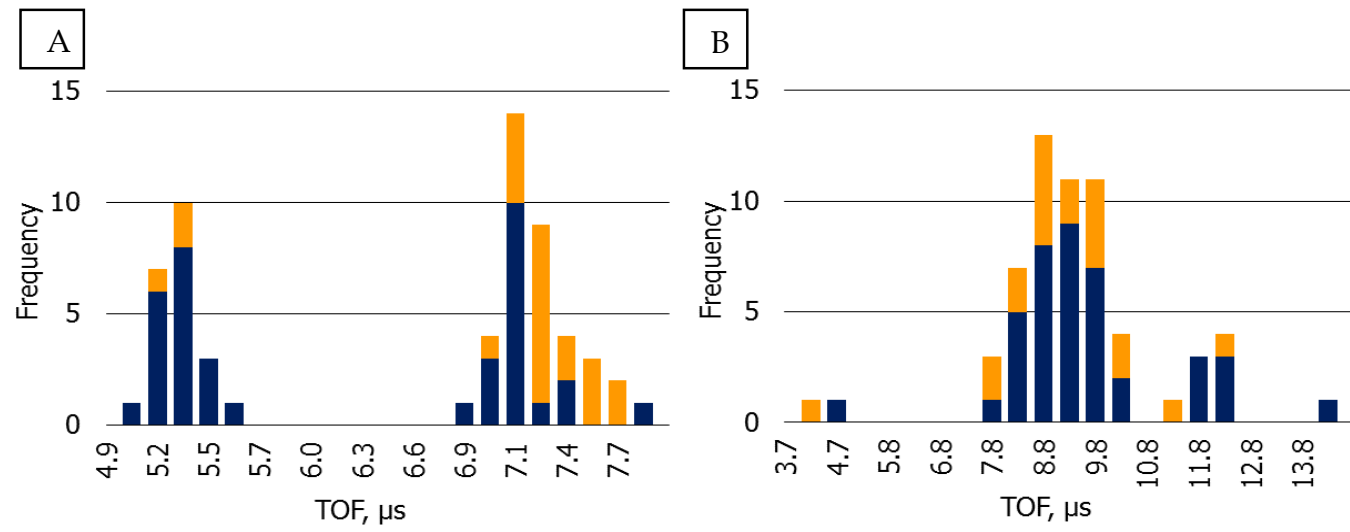

Fig. 6. Histograms of the TOF values for smoked $(\square)$ and untreated $(\square$ samples calculated via cross-correlation (A) and via the AIC picker method (B)

Table 1. Classification matrix using sample thickness and the AIC picker TOF as variables

\begin{tabular}{|c|c|c|c|c|c|}
\hline \multicolumn{2}{|c|}{} & \multicolumn{2}{|c|}{ Predicted Group Membership } & \multirow{2}{*}{ Total } \\
\cline { 3 - 5 } & \multirow{2}{|c|}{} & untreated & smoked & \\
\hline \multirow{3}{*}{$\begin{array}{c}\text { Original } \\
\text { Group } \\
\text { Membership }\end{array}$} & Count & untreated & 33 & 7 & 40 \\
\cline { 3 - 6 } & & smoked & 10 & 10 & 20 \\
\cline { 3 - 6 } & & untreated & 82.5 & 17.5 & 100.0 \\
\cline { 3 - 6 } & smoked & 50.0 & 50.0 & 100.0 \\
\hline
\end{tabular}

With a method applied in an earlier study using the original spectral data exclusively, that was gained via Fast Fourier Transformation (FFT) of the cross-correlated input and response signals, this ratio increased to $96.7 \%$ (CV: 66.7\%).

Table 2. Classification matrix using 66 FFT coefficients of the cross-correlated signals as variables

\begin{tabular}{|c|c|c|c|c|c|}
\hline \multicolumn{2}{|c|}{} & \multicolumn{2}{|c|}{ Predicted Group Membership } & \multirow{2}{*}{ Total } \\
\cline { 3 - 5 } & untreated & smoked & \\
\hline \multirow{3}{*}{$\begin{array}{c}\text { Original } \\
\text { Group } \\
\text { Membership }\end{array}$} & Count & untreated & 39 & 1 & 40 \\
\cline { 3 - 6 } & & smoked & 1 & 19 & 20 \\
\cline { 3 - 6 } & & untreated & 97.5 & 2.5 & 100.0 \\
\cline { 3 - 6 } & smoked & 5.0 & 95.0 & 100.0 \\
\hline
\end{tabular}


Through the wavelet-decomposition based approach described in this paper, this ratio increased to $100 \%$ (CV: $53.3 \%)$.

Table 3. Classification matrix using 65 wavelet coefficients of the cross-correlated signals as variables

\begin{tabular}{|c|c|c|c|c|c|}
\hline \multicolumn{2}{|c|}{} & \multicolumn{2}{|c|}{ Predicted Group Membership } & \multirow{2}{*}{ Total } \\
\cline { 3 - 5 } & untreated & smoked & \\
\hline \multirow{3}{*}{$\begin{array}{c}\text { Original } \\
\text { Group } \\
\text { Membership }\end{array}$} & Count & untreated & 40 & 0 & 40 \\
\cline { 3 - 6 } & & smoked & 0 & 20 & 20 \\
\cline { 3 - 6 } & & untreated & 100.0 & 0.0 & 100.0 \\
\cline { 3 - 6 } & smoked & 0.0 & 100.0 & 100.0 \\
\hline
\end{tabular}

\section{Conclusions}

The STA/LTA approach - although literature claims it can be very sensitive in case of optimal tuning (TRNKOCZY 2012) - is inapt for applications involving high levels of noise, including ultrasonic testing. In case of further refinement of noise-reduction, the method may be useful because of its sensitivity to parameter-adjustment. A possibly prosperous attempt to increase robustness could be to use the characteristic function as signal input of the STA/LTA function, as this was successfully used to increase robustness for the AIC picker method (SEDLAK et al. 2008).

The AR-AIC picker proved to be a useful tool, and a very robust method, offering reasonably reliable results for noisy or low-amplitude signals as well, which is an extremely important aspect in ultrasonic measurements. Calculating the AIC picker to different characteristic functions, or other fitted models with different key features such as the Multivariate Locally Stationary Autoregressive model (MLSAR) (TAKANAMI\&KITAGAWA 1991) could be a great way to increase the estimation of the spread of the signal. With that being said, processing time of the functions has to be evaluated to assess industrial relevance.

The best results during the experiment were achieved with wavelet analysis, which cannot characterize the dispersion of the signal per se, but with proper manipulations this information can be retained with sufficient amount of useful data. The result of the analysis was $100 \%$ correct classification, with samples being different only in processing methods and texture. 


\section{References}

ALLEN, R. (1982): Automatic phase pickers: Their present use and future prospects. Bulletin of the Seismological Society of America, 72, 225-242.

Benedito, J., CÁrcel, J., Clemente, G. \& Mulet, A. (2000a): Cheese maturity assessment using ultrasonics. Journal of Dairy Science, 83, 248-254.

Benedito, J., CÁrCel, J., SAnjuan, N. \& Mulet, A. (2000b): Use of ultrasound to assess Cheddar cheese characteristics. Ultrasonics, 38, 727-730.

Benedito, J., Simal, S., Clemente, G. \& Mulet, A. (2006): Manchego cheese texture evaluation by ultrasonics and surface probes. International Dairy Journal, 16, 431438.

Corredig, M., Alexander, M. \& Dalgleish, D. G. (2004): The application of ultrasonic spectroscopy to the study of the gelation of milk components. Food Research International, 37, 557-565.

DAUBECHIES, I. (1994): Ten lectures on wavelets. CBMS, SIAM, 61, 194-202.

Häupler, M., Peyronel, F., Neeson, I., Weiss, J. \& Marangoni, A. G. (2014): In situ ultrasonic characterization of cocoa butter using a chirp. Food and Bioprocess Technology, 7, 3186-3196.

LEEMANS, V. \& DESTAIN, M.-F. (2009): Ultrasonic internal defect detection in cheese. Journal of Food Engineering, 90, 333-340.

LÉtANG, C., PiAU, M., Verdier, C. \& LeFEbvre, L. (2001): Characterization of wheat-flourwater doughs: a new method using ultrasound. Ultrasonics, 39, 133-141.

MizRACH, A. (2004): Assessing plum fruit quality attributes with an ultrasonic method. Ultrasonics Sonochemistry, 18, 627-631.

Mizrach, A., Flitsanov, U., El-BatsRi, R. \& Degani, C. (1999): Determination of avocado maturity by ultrasonic attenuation measurements. Scientia Horticulturae, 80, 173-180.

Mizrach, A., Galili, N., Gan-Mor, S., Flitsanov, U. \& Prigozin, I. (1996): Model of ultrasonic parameters to assess avocado properties and shelf life. Journal of Agricultural Engineering Research, 65, 261-267.

SedlaK, P., Hirose, Y., A., ENOKI, M. \& SiKUlA, J. (2008): Arrival time detection in thin multilayer plates on the basis of akaike information criterion. EWGAE 166-171. Krakow.

TAKANAMI, T. \& KITAGAWA, G. (1991): Estimation of the arrival times of seismic waves by multivariate time series model. Annals of the Institute of Statistical Mathematics, 43 (3), 407-433.

TRNKOCZY, A. (2012): Understanding and parameter setting of STA/LTA trigger algorithm, BormAnN, P. (Ed.), New Manual of Seismological Observatory Practice 2, Potsdam, Germany, Deutsches GeoForschungsZentrum GFZ, 1-20. 